\title{
QSAR Study of the Reduction of Nitroaromatics by Fe(II) Species
}

\author{
DALIZZA Colón,* ERIC J. Weber AND JAMES L. ANDERSON
}

U.S. Environmental Protection Agency, National Exposure Research Laboratory, 960 College Station Road, Athens, Georgia 30605-27201

Department of Chemistry, University of Georgia, Athens, Georgia 30602-2556 ${ }^{2}$

Environmental Science \& Technology

SUPPORTING INFORMATION

May 2006

13 Pages

3 Figures

3 Tables 


\section{Supporting Information}

Anoxic Glove Box. Integrity of oxygen-sensitive suspensions, solutions and chemicals was preserved by storing them and performing all procedures involving them inside an anoxic glove box (Coy Lab) filled with $95 \% \mathrm{~N}_{2}: 5 \% \mathrm{H}_{2}$ at $21 \pm 1{ }^{\circ} \mathrm{C}$. The atmosphere of the glove box was constantly recirculated through two Pd catalyst barriers to scavenge oxygen. The oxygen and hydrogen levels inside the glove box were monitored with a meter (Coy Lab). Humidity inside the chamber was around 30\%. To transfer items inside and outside the glove box, the interlock chamber was evacuated to $-29 \mathrm{in}$. $\mathrm{Hg}$ and refilled with nitrogen three times. A minimum of a 20min period was allowed for oxygen depletion, whenever items were brought inside or taken outside the chamber, before exposing chemicals to the atmosphere of the glove box.

Synthesis of Mineral Phases. Goethite $(\alpha-\mathrm{FeOOH})$ was prepared by the slow addition of $2.2 \mathrm{~L}$ of $0.26 \mathrm{M} \mathrm{Fe}\left(\mathrm{NO}_{3}\right)_{3} \cdot 9 \mathrm{H}_{2} \mathrm{O}$ in $0.1 \mathrm{~N} \mathrm{HNO}_{3}$ into $5.0 \mathrm{~L}$ of $0.73 \mathrm{M} \mathrm{KOH}$ solution at $10{ }^{\circ} \mathrm{C}$. The resulting solid phase was heated at $70{ }^{\circ} \mathrm{C}$ for $24 \mathrm{~h}(1,2)$. Hematite $\left(\alpha-\mathrm{Fe}_{2} \mathrm{O}_{3}\right)$ was produced by vigorously mixing, at room temperature, $16.6 \mathrm{~g}$ of $\mathrm{Fe}\left(\mathrm{NO}_{3}\right)_{3} \cdot 9 \mathrm{H}_{2} \mathrm{O}$ with two $\mathrm{L}$ of $0.0002 \mathrm{M}$ $\mathrm{HNO}_{3}$, which had been heated to $98{ }^{\circ} \mathrm{C}$ in an oven. The reaction mixture was heated at $98{ }^{\circ} \mathrm{C}$ in an oven for seven days. Lepidocrocite $(\gamma-\mathrm{FeOOH})$ was made by adjusting the $\mathrm{pH}$ of a $0.20 \mathrm{M}$ $\mathrm{FeCl}_{2} \bullet 4 \mathrm{H}_{2} \mathrm{O}$ solution to 6.4 to 6.8 with $1 \mathrm{~N} \mathrm{NaOH}$ while it was purged with air at $280 \mathrm{~mL} / \mathrm{min}$. The synthesis was finished when the $\mathrm{pH}$ remained constant (3). 2-Line Ferrihydrite, $\sim \mathrm{Fe}(\mathrm{OH})_{3}$, was formed by rapidly raising the $\mathrm{pH}$ of one $\mathrm{L}$ of $0.1 \mathrm{M} \mathrm{FeCl}_{3} \bullet 6 \mathrm{H}_{2} \mathrm{O}$ solution by the addition, while stirring, of $160 \mathrm{~mL}$ of a $0.5 \mathrm{M} \mathrm{KOH}$ solution in the absence of air. The final $\mathrm{pH}$ of the suspension was 7.24. The product of each synthesis was decanted and frozen, followed by freeze-drying at $-70{ }^{\circ} \mathrm{C}$ and $8 \mu \mathrm{m} \mathrm{Hg}$. 
The identities of goethite, hematite and lepidocrocite were verified by X-ray powder diffraction analysis. The surface area analysis was performed by the BET method (4) using a Micromeritics ASAP2000 (Micromeritics Inc.) surface area analyzer.

Chemicals. Nanopure water, $\geq 18 \mathrm{M} \Omega$, was used for all experimental solution/suspension preparations. Degassed water was prepared by first boiling $900 \mathrm{~mL}$ of it for $20 \mathrm{~min}$ under argon, followed by sparging with argon for four additional $\mathrm{h}$. The remaining oxygen concentration in degassed water was assessed, with the Indigo Carmine method (using Accu Vac Ampules from Hach Co.), as statistically not different from zero (i.e., $1 \pm 1 \mu \mathrm{g} / \mathrm{L}$ ).

$p$ - $N$-hydroxylaminobenzonitrile (CNHA) was prepared by the addition of zinc and ammonium chloride to a stirred solution of CNNB in acetone (5). CNHA was purified by extracting it with ether and crystallized twice from ether to give yellow crystals. The product was stored in the dark under nitrogen.

The following chemicals were purchased from Aldrich, unless otherwise noted: nitrosobenzene (HNO), p-cyanonitrobenzene (CNNB), p-acetylnitrobenzene (AcNB), $p$ chloronitrobenzene (CINB), $p$-bromonitrobenzene (BrNB), nitrobenzene (HNB, ChemServices), p-nitrotoluene (MeNB), p-cyanoaniline (CNAN), $p$-aminoacetophenone (AcAN), $p$-chloroaniline (ClAN), $p$-bromoaniline (BrAN), aniline (HAN, Fluka) and $p$-toluidine (MeAN).

Reduction Experiments. Further reduction studies of CNNB were performed in individual Fe(II)-treated hematite $\left(34 \mathrm{~m}^{2} / \mathrm{g}\right)$, goethite $\left(51 \mathrm{~m}^{2} / \mathrm{g}\right)$, lepidocrocite $\left(49 \mathrm{~m}^{2} / \mathrm{g}\right)$ and ferrihydrite $\left(292 \mathrm{~m}^{2} / \mathrm{g}\right)$ suspensions as a function of their surface area loading (SAL): $0.55-60 \mathrm{~m}^{2} / \mathrm{L}, 1.0-112$ $\mathrm{m}^{2} / \mathrm{L}, 1.0-117 \mathrm{~m}^{2} / \mathrm{L}$, and $5.9-642 \mathrm{~m}^{2} / \mathrm{L}$, respectively. The effect of $\mathrm{pH}$ on the reduction of CNNB was evaluated in Fe(II)-treated hematite, goethite, lepidocrocite and ferrihydrite suspensions at the following specific conditions: $1.3 \mathrm{~m}^{2} / \mathrm{L}$ ferric oxide, $25 \mathrm{mM}$ sulfonic acid buffer ( $\mathrm{pH} 6.1$ to 
7.97) and $0.1 \mathrm{M} \mathrm{NaCl}$. Forty-eight hours were allowed for the equilibration of $\mathrm{Fe}(\mathrm{II})$ in the various oxide suspensions. In addition, the effect of the presence and concentration of NOM on $k_{\mathrm{CNNB}}$ was studied by varying the initial aqueous concentration of SRHA from 0 to $50 \mathrm{ppm}$ organic carbon (6) in $\mathrm{Fe}(\mathrm{II})$-treated goethite suspensions $\left(28 \mathrm{~m}^{2} / \mathrm{L}\right.$ goethite, $\mathrm{pH} 6.6$ and $0.1 \mathrm{M}$ $\mathrm{NaCl}$ ). $\mathrm{Fe}(\mathrm{II})$ was added $24 \mathrm{~h}$ after SRHA had been equilibrated in the suspension. NOMcontaining reactors were protected from light.

Molecular Modeling Calculations. The Gaussian 98 software package was used to calculate the energy of the lowest-unoccupied molecular orbital $\left(E_{\mathrm{LUMO}}\right)$. The calculations were performed with Hartree Fock and density functional (B3LYP) theory using the basis sets 6-31G* in the presence and absence of solvation, which was introduced using the PCM model.

Analysis of Organic Compounds. Sample aliquots (100 $\mu \mathrm{L})$ from kinetic experiments were analyzed by LC to separate, quantify and identify the components of interest (i.e., nitrobenzenes and related species). The Hewlett Packard 1050 LC was equipped with an autosampler, a quaternary pump, a diode array detector and a guard column (Alltech Adsorbosphere C18, $7.5 \mathrm{x}$ $4.6 \mathrm{~mm}, 5 \mu \mathrm{m}$ ) preceding an analytical column (Waters Symmetry C18, 150 x 4.6, $5 \mu \mathrm{m}$ ). The LC was used in the isocratic mode having the mobile phase made up of mixtures, anywhere from 19:81 to 40:60, of acetonitrile (J.T. Baker) and a pH 6.6 degassed 20-mm phosphate buffer $\left(\mathrm{K}_{2} \mathrm{HPO}_{4}\right.$ and $\mathrm{KH}_{2} \mathrm{PO}_{4}$, J.T. Baker); and the flow rate set at $1.0 \mathrm{~mL} / \mathrm{min}$.

$\mathbf{F e}(\mathbf{I I})$. Stock solutions of $\mathrm{Fe}(\mathrm{II})$ were prepared by mixing $0.15 \mathrm{~mL}$ of $1.0 \mathrm{M} \mathrm{HCl}$ (prepared from Trace Metal Grade, Fisher) with a $1.1 \mathrm{M}$ Fe(II) solution, which was prepared by dissolving $\mathrm{FeCl}_{2} \bullet 4 \mathrm{H}_{2} \mathrm{O}$ (Fluka) in water. To measure the $\mathrm{Fe}(\mathrm{III})_{\mathrm{aq}}$ content in samples, aliquots were withdrawn from the serum bottles with plastic syringes (no needles attached), filtered through $0.02 \mu \mathrm{m}$ Anotop syringe filter units and acidified to $\mathrm{pH} 1.4$ with $\mathrm{HCl}$ (Trace Metal Grade, 
Fisher). The Ferrozine method (7) was used to quantify Fe(II) $)_{\text {aq }}$. Our adaptation of this method involved mixing $37.5 \mu \mathrm{L}$ of the treated aliquot with $1.25 \mathrm{~mL}$ of $1.0 \mathrm{mM}$ Ferrozine (3-(2-pyridyl)5,6-bis(4-phenylsulfonic acid)-1,2,4-triazine monosodium salt monohydrate from Fluka) solution prepared in $0.5 \mathrm{M}$ MOPS buffer $(\mathrm{pH} 7.0)$. The concentration of $\mathrm{Fe}(\mathrm{II})_{\mathrm{aq}}$ was assessed by measuring the absorbance of the solution at $562 \mathrm{~nm}$ in a Hewlett Packard spectrophotometer (model 8453). 
TABLE S1. Effect of Ferric Oxide Surface Area Loading on the Rate of Reduction of p-Cyanonitrobenzene in Fe(II)-Treated Oxide Suspensions

\begin{tabular}{cccccc}
$\mathrm{SAL}\left(\mathrm{m}^{2} / \mathrm{L}\right)$ & $k(1 / \mathrm{h})^{a}$ & \multicolumn{5}{c}{$\mathrm{SD}^{\mathrm{b}}$} & $k / \mathrm{SAL}\left(\mathrm{m}^{2} / \mathrm{h} \cdot \mathrm{L}\right)$ & $\mathrm{Fe}(\mathrm{II})_{\text {surf,o }}{ }^{c}(\mu \mathrm{M})$ & $\mathrm{E}_{\mathrm{h}}(\mathrm{V})^{d}$ \\
& \multicolumn{5}{c}{ Goethite $\left(51 \mathrm{~m}^{2} / \mathrm{L}\right)$} \\
1.03 & $5.52 \mathrm{E}-03$ & $2.6 \mathrm{E}-04$ & $5.36 \mathrm{E}-03$ & 6.9 & 0.0109 \\
5.61 & $4.80 \mathrm{E}-01$ & $1.6 \mathrm{E}-03$ & $8.56 \mathrm{E}-02$ & 9.0 & 0.00400 \\
11.2 & $3.16 \mathrm{E}+00$ & $8.2 \mathrm{E}-02$ & $2.82 \mathrm{E}-01$ & 21 & -0.0178 \\
28.1 & $1.08 \mathrm{E}+01$ & $2.0 \mathrm{E}-01$ & $3.86 \mathrm{E}-01$ & 53 & -0.0417 \\
56.1 & $2.89 \mathrm{E}+01$ & $6.1 \mathrm{E}-01$ & $5.15 \mathrm{E}-01$ & 101 & -0.0583 \\
84.2 & $4.52 \mathrm{E}+01$ & $1.7 \mathrm{E}+00$ & $5.37 \mathrm{E}-01$ & 142 & -0.0669 \\
112 & $6.76 \mathrm{E}+01$ & $4.0 \mathrm{E}+00$ & $6.03 \mathrm{E}-01$ & 184 & -0.0736 \\
\multicolumn{5}{c}{ Lepidocrocite $\left(49 \mathrm{~m}^{2} / \mathrm{L}\right)$} \\
0.990 & $2.64 \mathrm{E}-03$ & $4.5 \mathrm{E}-05$ & $2.67 \mathrm{E}-03$ & 1.4 & 0.129 \\
5.87 & $2.64 \mathrm{E}-01$ & $4.1 \mathrm{E}-03$ & $4.50 \mathrm{E}-02$ & 13 & 0.0730 \\
11.7 & $9.38 \mathrm{E}-01$ & $5.8 \mathrm{E}-02$ & $7.99 \mathrm{E}-02$ & 22 & 0.0594 \\
29.4 & $2.37 \mathrm{E}+00$ & $2.1 \mathrm{E}-02$ & $8.06 \mathrm{E}-02$ & 56 & 0.0351 \\
58.7 & $3.20 \mathrm{E}+00$ & $2.5 \mathrm{E}-02$ & $5.45 \mathrm{E}-02$ & 105 & 0.0188 \\
88.1 & $3.60 \mathrm{E}+00$ & $4.2 \mathrm{E}-02$ & $4.09 \mathrm{E}-02$ & 152 & 0.00947 \\
117 & $3.37 \mathrm{E}+00$ & $5.9 \mathrm{E}-03$ & $2.87 \mathrm{E}-02$ & 193 & 0.00330 \\
\multicolumn{5}{c}{$\mathrm{Hematite}\left(34 \mathrm{~m}^{2} / \mathrm{L}\right)$} \\
0.552 & $6.24 \mathrm{E}-04$ & $4.4 \mathrm{E}-05$ & $1.13 \mathrm{E}-03$ & 2.4 & -0.156 \\
0.829 & $1.83 \mathrm{E}-03$ & $4.5 \mathrm{E}-04$ & $2.21 \mathrm{E}-03$ & 6.4 & -0.181 \\
1.11 & $4.10 \mathrm{E}-02$ & $3.8 \mathrm{E}-04$ & $3.71 \mathrm{E}-02$ & 6.9 & -0.183 \\
6.02 & $2.28 \mathrm{E}+00$ & $4.7 \mathrm{E}-02$ & $3.79 \mathrm{E}-01$ & 11 & -0.195 \\
12.0 & $9.92 \mathrm{E}+00$ & $8.2 \mathrm{E}-01$ & $8.24 \mathrm{E}-01$ & 12 & -0.197 \\
30.1 & $2.21 \mathrm{E}+01$ & $2.4 \mathrm{E}+00$ & $7.36 \mathrm{E}-01$ & 27 & -0.218 \\
60.2 & $6.07 \mathrm{E}+01$ & $4.0 \mathrm{E}+00$ & $1.01 \mathrm{E}+00$ & 37 & -0.227 \\
5.90 & $1.48 \mathrm{E}-04$ & $3.2 \mathrm{E}-05$ & $2.51 \mathrm{E}-05$ & 3.1 & 0.182 \\
32.1 & $2.69 \mathrm{E}-02$ & $2.3 \mathrm{E}-05$ & $8.37 \mathrm{E}-04$ & 3.2 & 0.182 \\
64.2 & $7.56 \mathrm{E}-02$ & $2.0 \mathrm{E}-03$ & $1.18 \mathrm{E}-03$ & 4.0 & 0.176 \\
161 & $2.41 \mathrm{E}-01$ & $1.2 \mathrm{E}-02$ & $1.50 \mathrm{E}-03$ & 9.2 & 0.155 \\
321 & $4.31 \mathrm{E}-01$ & $1.3 \mathrm{E}-02$ & $1.34 \mathrm{E}-03$ & 24 & 0.130 \\
482 & $8.25 \mathrm{E}-01$ & $1.2 \mathrm{E}-02$ & $1.71 \mathrm{E}-03$ & 34 & 0.121 \\
642 & $9.31 \mathrm{E}-01$ & $5.8 \mathrm{E}-05$ & $1.45 \mathrm{E}-03$ & 43 & 0.115
\end{tabular}

${ }^{a}$ Average pseudo-first order rate constant calculated from the linear regression of the concentration data ( $\mathrm{Ln}[\mathrm{CNNB}] /[\mathrm{CNNB}]_{\mathrm{o}}$ ) versus time (h) for duplicate experiments. ${ }^{b}$ Calculated standard deviation. ${ }^{c}$ Initial concentration of $\mathrm{Fe}$ (II) associated with the oxides surfaces. ${ }^{d}$ Calculated reduction potential for the Fe(II)/ferric oxide redox couple using the solubility product constant of the bulk oxide. Initial conditions: $15 \mu \mathrm{M} \mathrm{CNNB}, 375 \mu \mathrm{M} \mathrm{Fe}(\mathrm{II})$, pH 6.6 and $0.1 \mathrm{M} \mathrm{NaCl}$. 
TABLE S2. Effect of $p H$ and type of oxide on the concentration of $F e(I I)_{s u r f, o}, E_{h}$ and the Rate of Reduction of $\boldsymbol{p}$-Cyanonitrobenzene in $\mathrm{Fe}$ (II)-Treated Oxide Suspensions ${ }^{a}$

\begin{tabular}{|c|c|c|c|c|c|}
\hline $\mathrm{pH}$ & $k^{b}(1 / \mathrm{h})$ & $\mathrm{SD}^{c}$ & $\begin{array}{c}k / \mathrm{SAL} \\
\left(\mathrm{m}^{2} / \mathrm{h} \cdot \mathrm{L}\right)\end{array}$ & $\begin{array}{c}\mathrm{Fe}(\mathrm{III})_{\text {surf,o }}{ }^{d} \\
\quad(\mu \mathrm{M})\end{array}$ & $\mathrm{E}_{\mathrm{h}}(\mathrm{V})^{e}$ \\
\hline \multicolumn{6}{|c|}{ Goethite } \\
\hline 7.97 & $8.33 \mathrm{E}+00$ & $5.0 \mathrm{E}-01$ & $6.41 \mathrm{E}+00$ & 31 & -0.271 \\
\hline 7.67 & $2.02 \mathrm{E}+00$ & $3.2 \mathrm{E}-02$ & $1.55 \mathrm{E}+00$ & 9.4 & -0.187 \\
\hline 7.50 & $7.95 \mathrm{E}-01$ & $2.4 \mathrm{E}-02$ & $6.11 \mathrm{E}-01$ & 18 & -0.173 \\
\hline 7.28 & $1.52 \mathrm{E}-01$ & $6.8 \mathrm{E}-03$ & $1.17 \mathrm{E}-01$ & 11 & -0.117 \\
\hline 7.00 & $2.43 \mathrm{E}-02$ & $1.3 \mathrm{E}-03$ & $1.87 \mathrm{E}-02$ & 8.4 & -0.0652 \\
\hline 6.80 & $1.52 \mathrm{E}-02$ & $1.3 \mathrm{E}-03$ & $1.17 \mathrm{E}-02$ & 0.44 & 0.0459 \\
\hline 6.60 & $5.52 \mathrm{E}-03$ & $2.6 \mathrm{E}-04$ & $4.25 \mathrm{E}-03$ & 1.8 & 0.0454 \\
\hline 6.10 & $2.77 \mathrm{E}-04$ & $6.4 \mathrm{E}-06$ & $2.13 \mathrm{E}-04$ & 7.6 & 0.0970 \\
\hline \multicolumn{6}{|c|}{ Lepidocrocite } \\
\hline 7.97 & $2.05 \mathrm{E}+01$ & $1.5 \mathrm{E}+00$ & $1.58 \mathrm{E}+01$ & 29 & -0.192 \\
\hline 7.67 & $3.82 \mathrm{E}+00$ & $2.4 \mathrm{E}-01$ & $2.94+00$ & 14 & -0.121 \\
\hline 7.50 & $1.12 \mathrm{E}+00$ & $6.0 \mathrm{E}-02$ & $8.61 \mathrm{E}-01$ & 27 & -0.106 \\
\hline 7.28 & 4.20E-01 & 2.3E-03 & $3.23 \mathrm{E}-01$ & 11 & -0.0387 \\
\hline 7.00 & $5.63 \mathrm{E}-02$ & $3.2 \mathrm{E}-03$ & 4.33E-02 & 11 & 0.00494 \\
\hline 6.80 & $1.80 \mathrm{E}-02$ & $3.1 \mathrm{E}-04$ & $1.39 \mathrm{E}-02$ & 13 & 0.0373 \\
\hline 6.60 & $2.64 \mathrm{E}-03$ & $4.5 \mathrm{E}-05$ & $2.03 \mathrm{E}-03$ & 1.5 & 0.129 \\
\hline 6.10 & $1.58 \mathrm{E}-04$ & $3.2 \mathrm{E}-07$ & $1.21 \mathrm{E}-04$ & 4.6 & 0.188 \\
\hline \multicolumn{6}{|c|}{ Hematite } \\
\hline 7.97 & $4.31 \mathrm{E}+01$ & $3.9 \mathrm{E}+00$ & $3.32 \mathrm{E}+01$ & 43 & -0.473 \\
\hline 7.67 & $1.83 \mathrm{E}+01$ & 8.2E-01 & $1.41 \mathrm{E}+01$ & 27 & -0.408 \\
\hline 7.50 & $5.29 \mathrm{E}+00$ & 4.0E-01 & $4.07 \mathrm{E}+00$ & 28 & -0.381 \\
\hline 7.28 & $1.11 \mathrm{E}+00$ & 4.3E-02 & $8.52 \mathrm{E}-01$ & 6.6 & -0.297 \\
\hline 7.00 & $4.88 \mathrm{E}-01$ & $6.0 \mathrm{E}-03$ & $3.75 \mathrm{E}-01$ & 4.3 & -0.242 \\
\hline 6.80 & $1.68 \mathrm{E}-01$ & $1.2 \mathrm{E}-02$ & $1.29 \mathrm{E}-01$ & 4.2 & -0.206 \\
\hline 6.60 & 4.10E-02 & 3.8E-04 & $3.15 \mathrm{E}-02$ & 6.9 & -0.183 \\
\hline 6.10 & $1.71 \mathrm{E}-03$ & 5.9E-05 & $1.31 \mathrm{E}-03$ & 0.25 & -0.00952 \\
\hline \multicolumn{6}{|c|}{ Ferrihydrite } \\
\hline 7.97 & $1.79 \mathrm{E}-01$ & $9.8 \mathrm{E}-03$ & $1.37 \mathrm{E}-01$ & 30 & -0.119 \\
\hline 7.67 & $3.09 \mathrm{E}-02$ & $1.0 \mathrm{E}-03$ & $2.38 \mathrm{E}-02$ & 7.9 & -0.0310 \\
\hline 7.50 & $7.13 \mathrm{E}-03$ & 4.6E-03 & $5.48 \mathrm{E}-03$ & 20 & 0.0244 \\
\hline 7.28 & 8.31E-04 & $1.6 \mathrm{E}-05$ & $6.39 \mathrm{E}-04$ & 12 & 0.0338 \\
\hline 7.00 & $5.97 \mathrm{E}-04$ & 4.9E-05 & $4.59 \mathrm{E}-04$ & 0.36 & 0.167 \\
\hline 6.80 & $1.92 \mathrm{E}-04$ & $2.8 \mathrm{E}-05$ & $1.47 \mathrm{E}-04$ & 2.4 & 0.154 \\
\hline 6.60 & $1.48 \mathrm{E}-04$ & $3.2 \mathrm{E}-05$ & $1.14 \mathrm{E}-04$ & 3.1 & 0.182 \\
\hline $6.1^{f}$ & & & & & \\
\hline \multicolumn{6}{|c|}{$\mathrm{Fe}(\mathrm{II})$} \\
\hline 7.97 & $1.91 \mathrm{E}-02$ & 2.7E-03 & & & \\
\hline 7.67 & $5.82 \mathrm{E}-03$ & $3.1 \mathrm{E}-04$ & & & \\
\hline 7.50 & $1.30 \mathrm{E}-03$ & 5.0E-05 & & & \\
\hline 7.28 & $2.37 \mathrm{E}-04$ & $9.8 \mathrm{E}-06$ & & & \\
\hline $7.00^{f}$ & & & & & \\
\hline
\end{tabular}


$6.80^{f}$

$6.60^{f}$

$6.10^{f}$

${ }^{a}$ Initial conditions: $15 \mu \mathrm{M} \mathrm{CNNB}, 1.3 \mathrm{~m}^{2} / \mathrm{L}$ ferric oxide, $375 \mu \mathrm{M}$ Fe(II), $25 \mathrm{mM}$ of sulfonic acid buffer (MES for 6.1 and 6.6, MOPSO for 6.8, MOPS for 7.0, 7.28 and 7.5 and EPPS for 7.67 and 7.97) and $0.1 \mathrm{M} \mathrm{NaCl} .{ }^{b}$ Average pseudo-first order rate constant calculated from the linear regression of the concentration data $\left(\mathrm{Ln}[\mathrm{CNNB}] /[\mathrm{CNNB}]_{\mathrm{o}}\right)$ versus time $(\mathrm{h})$ for duplicate experiments. ${ }^{c}$ Calculated standard deviation. ${ }^{d}$ Initial concentration of $\mathrm{Fe}(\mathrm{II})_{\mathrm{aq}}$ lost from solution during the equilibration time. ${ }^{e}$ Calculated reduction potential for the $\mathrm{Fe}$ (II)/ferric oxide redox couple using the solubility product constant of the bulk oxide. ${ }^{f}$ No reaction was observed. 


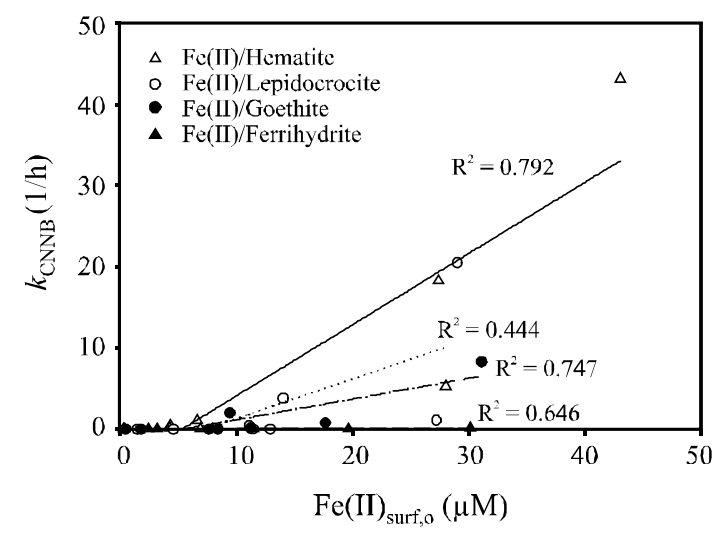

Figure S1. Dependence of the reduction rate constant of $\mathrm{CNNB}$ on $\mathrm{Fe}(\mathrm{II})_{\text {surf,o }}$ in $\mathrm{Fe}$ (II)treated hematite (-), goethite (.-.), lepidocrocite (..) and ferrihydrite (---) suspensions in a pH-variation experiments. Initial conditions: $15 \mu \mathrm{M} \mathrm{CNNB}, 375 \mu \mathrm{M} \mathrm{Fe}(\mathrm{II}), 1.3 \mathrm{~m}^{2} / \mathrm{L}$ ferric oxide, $25 \mathrm{mM}$ sulfonic acid buffer and $0.1 \mathrm{M} \mathrm{NaCl}$. 

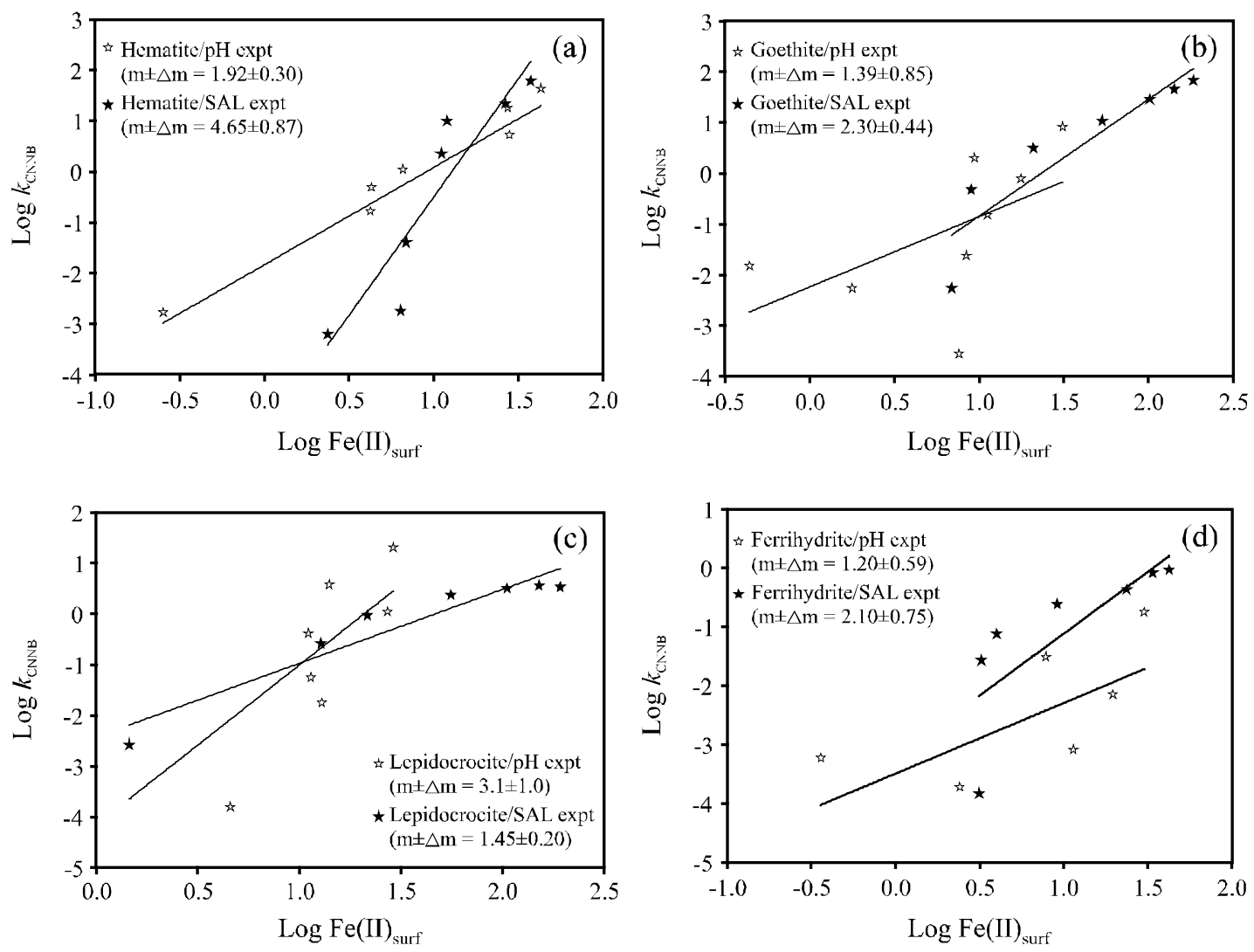

Figure S2. Apparent reaction-order plot for the reduction of CNNB by surface-associated $\mathrm{Fe}$ (II) in Fe(II)-treated (a) hematite, (b) goethite, (c) lepidocrocite and (d) ferrihydrite suspensions as a function of $\mathrm{pH}$ and $\mathrm{SAL}$ variations. Lines are linear regressions. 


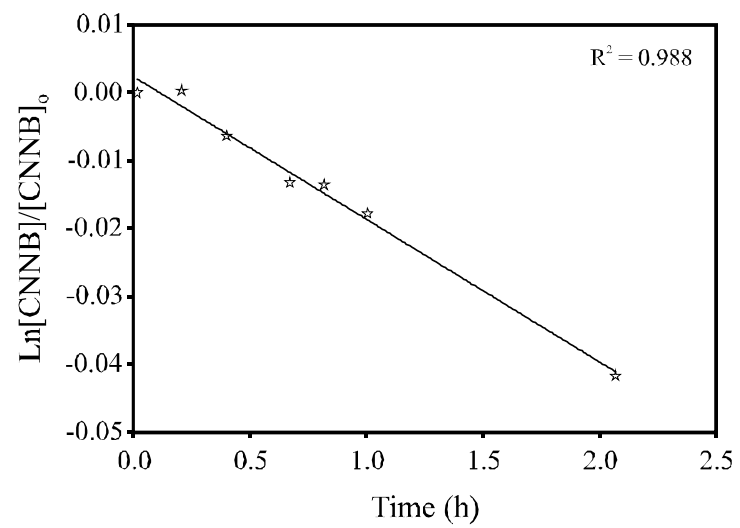

Figure S3. Pseudo-first order plot for the reduction of CNNB in Fe(II) solution at pH 7.97. Initial conditions: $15 \mu \mathrm{M}$ CNNB, $375 \mu \mathrm{M}$ Fe(II), 25 mM MES EPPS and 0.1 M NaCl. 
TABLE S3. Literature values for Solubility Product Constants used in $\mathbf{E}_{\mathbf{h}}$ calculations

\begin{tabular}{|c|c|}
\hline Species & Log Solubility Product ${ }^{a}$ \\
\hline Ferrihydrite & -38.04 \\
\hline Goethite & -40.6 \\
\hline Hematite & -43.88 \\
\hline Lepidocrocite & -39.28 \\
\hline
\end{tabular}

${ }^{a}$ Reference (8).

\section{Example of a calculation of Kso derived $E_{h}$}

$\mathrm{Fe}^{2+}=\mathrm{Fe}^{3+}+\mathrm{e}^{-}$ $\mathrm{E}^{\circ}=0.770$ volts

$\alpha-\mathrm{FeOOH}+\mathrm{H}_{2} \mathrm{O}=\mathrm{Fe}^{3+}+3 \mathrm{OH}^{-}$ $\log$ Kso $=-40.6$

$\gamma_{\mathrm{Fe}(\mathrm{II})}=0.40$ at $0.1 \mathrm{M}$ ionic strength* (9).

*This value is valid at $25^{\circ} \mathrm{C}$, we are working under the assumption that the difference between $\gamma_{\mathrm{Fe}(\mathrm{II})}$ at $21^{\circ} \mathrm{C}$ and $25^{\circ} \mathrm{C}$ is negligible.

Surface area loading experiment for goethite at $84.15 \mathrm{~m}^{2} / \mathrm{L}$ :

Ionic strength

$\mathrm{a}_{\mathrm{Fe}(\mathrm{II})}=$ molarity $*$ activity coefficient

$\mathrm{E}=\mathrm{E}^{\circ}+\mathrm{RT} / \mathrm{nF} \ln \mathrm{a}_{\mathrm{Fe}(\mathrm{III})} / \mathrm{a}_{\mathrm{Fe}(\mathrm{II})}$

$\mathrm{E}=0.77+\mathrm{RT} / \mathrm{nF} \ln (\mathrm{Kso})\left(\mathrm{a}_{\mathrm{H}}^{+}\right)^{3} /(\mathrm{Kw})^{3} \mathrm{a}_{\mathrm{Fe}(\mathrm{II})}$

$\mathrm{E}=0.77+\mathrm{RT} / \mathrm{nF} \ln (2.51 \mathrm{e}-41)(2.51 \mathrm{e}-07)^{3} /(10 \mathrm{e}-14)^{3}(5.83 \mathrm{e}-05)$

$\mathrm{E}=-0.0669$ volts

\section{$0.1 \mathrm{M} \mathrm{NaCl}$}

$(1.42 \mathrm{e}-04)^{*} 0.40=5.68 \mathrm{e}-05$ 


\section{Literature Cited}

(1) Atkinson, R. J.; Posner, A. M.; Quirk, J. P. Adsorption of potential-determining ions at the ferric oxide-aqueous electrolyte interface. J. Phys. Chem. 1967, 71, 550-558.

(2) Torrents, A.; Stone, A. T. Hydrolysis of phenyl picolinate at the mineral/water interface. Environ. Sci. Technol. 1991, 25, 143-149.

(3) Schwertmann, U.; Cornell, R. M. Iron Oxides in the Laboratory: Preparation and Characterization; VCH: New York, 1991.

(4) Brunauer, S.; Emmett, P. H.; Teller, E. J. Am. Chem. Soc 1938, 60.

(5) Fletcher, D. A. Structural investigations of $C$-nitrosobenzenes. Part 1. Solution state ${ }^{1} \mathrm{H}$ NMR studies. J. Chem. Soc. Perk. Trans. 1997, 2, 2201-2206.

(6) Senesi, N.; Miano, T. M.; Provenzano, M. R.; Brunetti, G. Spectroscopic and Compositional Comparative Characterization of IHSS Reference and Standard Fulvic and Humic Acids of Various Origins. Sci. Total. Environ. 1989, 81/82, 143-156.

(7) Strathmann, T. J.; Stone, A. T. Reduction of the carbamate pesticides oxamyl and methomyl by dissolved Fe ${ }^{\mathrm{II}}$ and $\mathrm{Cu}^{\mathrm{I}}$. Environ. Sci. Technol. 2001, 35, 2461-2469.

(8) Cornell, R. M.; Schwertman, U. The Iron Oxides: Structure, Properties, Reactions, Occurrences and Uses; Second ed.; Wiley-VCH: Weinheim, 2003.

(9) Skoog, D. A.; West, D. M. Fundamentals of Analytical Chemistry; Fourth ed.; Saunders: Philadelphia, 1982. 